\title{
Análisis de desigualdades en salud. Una propuesta cualitativa*
}

\author{
Analysis of health inequalities. A qualitative approach
}

\author{
Análise de desigualdades em saúde. Uma proposta qualitativa
}

\begin{abstract}
Nicolás Ortiz Ruiz*
* Magíster en Salud Pública, magíster en Ciencias Sociales, con énfasis en Sociología de la Modernización, doctor en Salud Colectiva. Profesor de la Escuela de Salud Pública de la Universidad del Valle, Cali (Colombia). nicolas.ortiz@correounivalle.edu.co, oRcid: https://orcid.org/0000-0001-8730-3773
\end{abstract}

Recibido: 23/07/2019. Aprobado: 22/05/2020 . Publicado: 30/10/2020

Ortíz-Ruíz N. Análisis de desigualdades en salud. Una propuesta cualitativa. Rev. Fac. Nac. Salud Pública. 2020;39(1):e339563. DoI: https://doi.org/10.17533/udea.rfnsp.e339563

\section{Resumen}

Objetivo: Presentar los resultados de un estudio de las desigualdades sociales a partir de experiencias de manejo y tratamiento de diabetes mellitus tipo 2, y aportar elementos teóricos y metodológicos para analizar las desigualdades sociales en salud. Metodología: Investigación cualitativa, basada en relatos biográficos de personas diagnosticadas hacía más de un año, y con características socioeconómicas y socioculturales diversas. Los casos fueron seleccionados en diferentes centros de salud del municipio de Santiago de Cali (Colombia), procurando tener representación de clase social, género y condición étnico-racial diversas. Resultados: Se identificaron tres ámbitos que, articulados entre sí, configuran las condiciones de desigualdad a lo largo de las trayectorias de vida y en las experiencias de manejo y tratamiento de la diabetes. Estos son: el ámbito de la salud, que incluye el acceso y uso de servicios de salud y la satisfacción de necesidades en salud, el cuidado de la salud y los resultados en salud; el ámbito socioeconómico/cultural, que incluye los espacios sociales, las posiciones sociales, los capitales y los esquemas socioculturales de clasificación-jerarquización social; por último, el ámbito del contexto, que incluye las políticas y las situaciones económicas, sociales y culturales del entorno. Conclusiones: Analizar las desigualdades en salud implica comprender su producción social y no simplemente sus manifestaciones en los cuerpos o el acceso a servicios de salud. Se identificaron cuatro ejes claves de producción de desigualdades: el territorio, la articulación entre educacióntrabajo, el género y las diferentes formas de violencia.

-Palabras clave: Desigualdades en salud, investigación cualitativa, condiciones sociales, determinantes sociales de la salud, servicios de salud.

* Tesis para optar al título de doctor en Salud Colectiva, Universidad Federal del Espirito Santo: "Desigualdades sociais em experiências de adoecimento por diabetes mellitus tipo 2", de Nicolás Ortiz Ruiz, 2019. 


\begin{abstract}
Objective: To present the findings of a study about social inequalities based on experiences of care and treatment of patients affected by type 2 Diabetes Mellitus and to provide theoretical and methodological elements to analyze social inequalities in health. Methodology: Qualitative study based on biographical stories of patients diagnosed for more than one year having different socioeconomic and sociocultural features was carried out. In an attempt to have representation of diverse social classes, genders and ethnic-racial conditions, cases were selected from different healthcare facilities of the Santiago de Cali municipality (Colombia). Results: Three interrelated domains which constitute conditions of inequality throughout life and in experiences of the care and treatment of diabetes were identified. They were health domain, which comprises the access
\end{abstract}

and use of health services and satisfaction of health needs, health care and health results; socioeconomic domain, which includes social spaces, social positions, resources, sociocultural frames of social hierarchy formation; and finally, context domain, which involves policies and economic, social and cultural features of the patient's environment. Conclusions: In order to analyze health inequalities, not only understanding their manifestations in organisms or access to health services is necessary but also the comprehension of their social development. Four key elements for the development of inequalities were identified: the territory, the link between education and employment, gender, and various forms of violence.

--------Keywords: Health inequalities, qualitative study, social conditions, social determinants of health, health services.

\section{Resumo}

Objetivo: Apresentar os resultados de uma pesquisa relacionada com as desigualdades sociais partindo de experiências de manejo e tratamento do Diabetes Mellitus Tipo 2, além de contribuir com elementos teóricos e metodológicos para analisar as desigualdades sociais em saúde. Metodologia: Pesquisa qualitativa, baseada em relatos biográficos de pessoas diagnosticadas há mais de um ano e com características socioeconômicas e socioculturais diversas. Os casos foram selecionados em diferentes centros de saúde do município de Santiago de Cali (Colômbia), procurando ter representação de classe social, gênero e condição étnico-racial diversas. Resultados: Foram identificadas três áreas que, combinadas entre si, formam as condições de desigualdade ao longo das experiências de vida e nos cuidados e tratamento do diabetes, que são: 1) Área da saúde, que inclui o acesso e uso de serviços de saúde e a satisfação de necessidades em saúde, o cuidado da saúde e os resultados em saúde; 2) Área social econômica/ cultural, que inclui os espaços sociais, as posições sociais, os capitais e os esquemas socioculturais de classificaçãohierarquia social; e finalmente, 3) Área do contexto, que inclui as políticas e as situações econômicas, sociais e culturais do entorno. Conclusões: Analisar as desigualdades em saúde significa compreender sua produção social e não simplesmente suas manifestações nos corpos ou o acesso aos serviços de saúde. Foram identificados quatro campos-chave geradores de desigualdades: o território, a articulação entre educação e trabalho, o gênero e as diferentes formas de violência.

-Palavras-chave: Desigualdade em saúde; pesquisa qualitativa; condições sociais; determinantes sociais da saúde; serviços de saúde

\section{Introducción}

Las desigualdades en salud son un problema global que afecta países pobres y ricos, cuya persistencia se constituye en uno de los problemas de salud pública más serios y desafiantes [1]. Estas desigualdades influyen de manera diferencial en los grupos, según condiciones socioeconómicas, de género, étnico/racial o de diversidad sexual, entre otras categorías sociales, en aspectos que tienen que ver con los resultados de salud, el acceso a servicios de salud, la calidad de la atención y la de las relaciones entre el personal de la salud y los pacientes $[2,3]$.
Metodológicamente, la tendencia más difundida para medir las desigualdades apela a métodos cuantitativos, siguiendo dos pasos: "1) la comparación del estado de salud de dos o más grupos poblacionales socialmente definidos y 2) la expresión de esa comparación en una métrica-resumen, específica y estándar, de la desigualdad en salud. Esta métrica-resumen puede expresar una brecha (la diferencia entre dos y solo dos grupos sociales, usualmente extremos) o bien un gradiente (la magnitud de la desigualdad en salud a lo largo de toda la jerarquía social), y puede ser absoluta [...] o bien relativa $[\ldots]$ " [4, p. 3]. En este marco, aquellos que ocupan posiciones más bajas en la escala socioeconómica exhiben peor estado de salud que quienes están por encima [5]. 
Con el propósito de explorar otros marcos de estudio diferente a los cuantitativos, se usaron métodos cualitativos para analizar los efectos de las desigualdades sociales en experiencias de pacientes con diabetes mellitus tipo 2, tomando en cuenta el incremento de la prevalencia en contextos de privación con bajo nivel de ingresos y bajo nivel educativo [6], así como el condicionamiento de la posición socioeconómica en los cuidados de la salud, la prevención de la enfermedad, las medidas de promoción de la salud, la disposición a buscar tratamiento y los estilos de vida [7-9].

En Colombia, según estimaciones de 2007, las mujeres tenían 1,35 veces más diabetes que los hombres; las personas sin educación, 1,86 veces que las que tenían educación superior, y las del quintil de menor riqueza, 2,1 veces que las del quintil 4 [10]. En el municipio de Cali, las muertes por diabetes ocupan el cuarto lugar en el grupo de enfermedades crónicas no transmisibles [11]. En Colombia, los ciudadanos tienen derecho a un paquete de servicios común para la atención de la diabetes, que incluye atención clínica, medicamentos y laboratorio clínico; no obstante, hay desigualdades, como se describe más adelante.

En este artículo, se presenta parte de los resultados de una investigación cualitativa realizada en el municipio de Santiago de Cali (Colombia), en 2018. Se propone aportar elementos teóricos y metodológicos para analizar las desigualdades sociales, a partir de trayectorias de vida, en las que confluyen las "pequeñas" historias biográficas con la gran historia social.

Los resultados parten de un marco teórico basado en el constructivismo estructural sobre la base de conceptos como habitus, posiciones sociales y espacios o escenarios sociales, para explorar las desigualdades sociales en las trayectorias de vida y en las experiencias de enfermar. La propuesta teórico-metodológica que se realiza recoge dichos elementos, pero añade otros, provenientes del análisis de los relatos de los participantes. De este modo, en la propuesta se articulan tres ámbitos de análisis de las desigualdades sociales: ámbito de la salud, ámbito socioeconómico/cultural y ámbito de contexto. Adicionalmente, emergen conceptos que no fueron considerados desde el inicio, como: rupturas biográficas y transiciones.

\section{Metodología}

Esta investigación se sitúa en el paradigma cualitativo y teóricamente se nutre del constructivismo-estructural, que postula que las realidades sociales son construcciones históricas y cotidianas de actores individuales y colectivos, es decir, no están bajo el control de la voluntad de los individuos. Bajo esta dinámica social, según el acceso/posesión de los individuos a diferentes formas de capital a lo largo de sus vidas y a determinadas condiciones de existencia, ocuparán ciertas posiciones sociales, que condicionan sus disposiciones, gustos, prácticas, esquemas de pensamiento, relaciones con su cuerpo y con los otros (habitus) [12].

En este marco, se analizan las desigualdades en salud, a partir del conocimiento de las experiencias de personas con diabetes, mediadas por condiciones socialmente estructuradas con respecto al acceso y la disponibilidad de oportunidades y recursos, así como de las formas de clasificación y división sociocultural. Se reconocen como premisas: 1) la distribución de la salud y la enfermedad en poblaciones no pueden ser comprendidas aparte de $-\mathrm{y}$ necesariamente ocurre en - su contexto social; 2) los procesos sociales históricamente producidos determinan y moldean el proceso salud/enfermedad/ cuidado, y 3) a medida que cambian las sociedades, en términos sociales, culturales, económicos o tecnológicos, también lo harán la distribución y los niveles de salud y enfermedad en la población [13]. ${ }^{\dagger}$

Metodológicamente, el investigador realizó directamente 23 entrevistas individuales durante el 2018, a personas con características socioeconómicas y socioculturales diversas. En promedio, las entrevistas duraron 1 hora y 30 minutos para acceder a los relatos biográficos, por medio de los cuales las personas narraron sus experiencias. Se implementó un instrumento para guiar cronológicamente los relatos, desde la infancia hasta el presente. Los ejes de indagación en cada etapa fueron: principales espacios sociales y experiencias más significativas a lo largo de sus vidas (espacios de socialización), ventajas y desventajas percibidas (materiales y no materiales), tipos y calidad de las relaciones sociales.

Todos los casos fueron seleccionados directamente en las consultas de los centros de salud del municipio de Santiago de Cali, buscando una representación diversa de clase social, género y condición étnico-racial, y que tuvieran diagnóstico de diabetes de más de un año. Asumir este tiempo de diagnóstico como el único criterio de inclusión obedeció a la probabilidad de incorporar a la investigación a personas que hubiesen experimentado cambios desde el momento del diagnóstico, relacionados con la aceptación/adaptación/resignificación de su vida y prácticas diarias, acceso y uso de servicios de sa-

En la premisa 2, el texto original dice "procesos sociales causales (aunque probabilísticos) determinan y moldean todos los desenlaces de salud y enfermedad". Este cambio responde a que no estoy de acuerdo con que sean causales, de carácter probabilísticos, pues parecería ser producto del azar. Considero que son históricamente producidos, en contextos sociales y bajo relaciones de poder específicas. 
lud, así como la posibilidad de experimentar la enfermedad en diferentes ámbitos de su vida.

El proyecto fue aprobado por el Comité de Ética de la Universidad Federal de Espirito Santo, de Brasil (Aprobación 2.201.909 de 2017), y contó con el aval de la Secretaría Municipal de Salud de Santiago de Cali. A los participantes les fue solicitado su consentimiento informado previo a la entrevista, tras presentarles los objetivos de la investigación; además, fueron informados sobre la confidencialidad de los datos suministrados y del anonimato de sus nombres.

Las participantes tienen entre 38 y 85 años, con una media de 60 años, de los cuales 14 son mujeres, 17 se reconocen como mestizos o mestizas, 5 como negros o negras, y solo una como blanca. Frente al diagnóstico, 10 de los participantes fueron diagnosticados: 2 , hace 4 años; 7, entre 5 y 15 años, y 6, hace más de 15 años. Respecto al nivel de formación, 6 cursaron primaria (completa e incompleta), 6 secundaria (completa e incompleta), 8 educación técnica y universitaria, y 3 no estudiaron. Por último, 12 pertenecen al régimen contributivo, 11 al régimen subsidiado del sistema general de seguridad social en salud ${ }^{ \pm}$y 12 viven en barrios de estratos 1 y $2 ; 8$ en 3 y 4, y 3 en $5 .{ }^{\S}$

Por otro lado, se realizó un análisis de contenido temático, esto es, un ejercicio sistemático de interpretación de la información recopilada, para identificar contenido explícito y latente, situado en los contextos de los informantes [16]. Se identificaron las unidades de registro, correspondientes a fragmentos de texto en los que se comunicó una idea relacionada con los objetivos de la investigación, y estas unidades, a su vez, se ubicaron en unidades de contextos más amplios, que permitieron su compresión con sentido [17].

Inicialmente, las unidades de registro se codificaron utilizando algún término de su contenido que las identificaba y las diferenciaba de otras, y luego los códigos se agruparon en categorías definidas desde el marco teórico. Por lo tanto, comenzó con una lógica inductiva, pero, más tarde, las unidades codificadas se enmarcaron en categorías teóricas. Algunas de las subcategorías no tenían un marco teórico previamente definido, lo que condujo a la creación de categorías que eran consistentes con el marco teórico. En la Tabla 1 se presentan las categorías y subcategorías resultantes.
Tabla 1. Categorías y subcategorías.

\begin{tabular}{|c|c|}
\hline Categorías & Subcategorías \\
\hline $\begin{array}{l}\text { Articulaciones } \\
\text { de desigualdad }\end{array}$ & $\begin{array}{l}\text { Encadenamiento educación-trabajo- } \\
\text { acceso a bienes y servicios } \\
\text { Encadenamiento territorio-acceso a } \\
\text { bienes y servicios } \\
\text { Redes-autocuidado } \\
\text { Estereotipos de género-autocuidado } \\
\text { Técnicas corporales-autocuidado }\end{array}$ \\
\hline Posición social & $\begin{array}{l}\text { Posición económica } \\
\text { Posición simbólica } \\
\text { Capitales (económico, social, cultural, } \\
\text { simbólico) }\end{array}$ \\
\hline
\end{tabular}

En el siguiente apartado, se presentan sintéticamente aspectos del contexto y de las trayectorias de vida y, con mayor detalle, combinado con algunas referencias teóricas, las experiencias de desigualdad en el manejo y el tratamiento de la diabetes, procurando articular en torno a ellas el contexto y las trayectorias de vida.

La presentación de los resultados se realiza a partir de la voz del investigador. Allí se muestran los principales hallazgos con base en las categorías, recuperando testimonios de los participantes, insumo fundamental de este estudio.

\section{Resultados}

Respecto al contexto, el país y el municipio de Cali tienen altos niveles de desigualdad, convirtiendo esto en una característica destacada, manifiesta en la distribución del ingreso, de la tierra, en la desigualdad de género, en la discriminación racial y étnica. En las entrevistas, fueron referenciados los siguientes aspectos del contexto:

- Procesos de migración forzada, por la violencia en las zonas rurales, así como por los procesos de industrialización y el desarrollo de servicios sociales en las ciudades.

- La violencia en ciudades, por narcotráfico.

- Violencia intrafamiliar y de estereotipos de género, que marcan desigualdades entre hombres y mujeres.

- Informalidad y precarización laboral.

- Desigualdad entre lo urbano y lo rural, entre ingresos, entre géneros y entre razas/etnias.

En Colombia, los ciudadanos acceden a los servicios de salud a través del "Régimen Contributivo", que incluye las personas que aportan mensualmente un porcentaje de sus ingresos como trabajadores dependientes o independientes, o del "Régimen Subsidiado", que corresponde a personas que no tienen capacidad de pago y, por tanto, acuden a servicios públicos. Para más detalles, véase [14].

\$ La estratificación socioeconómica en Colombia se refiere a la clasificación de los inmuebles residenciales que deben recibir servicios públicos. "los estratos 1, 2 y 3 corresponden a estratos bajos que albergan a los usuarios con menores recursos, los cuales son beneficiarios de subsidios en los servicios públicos domiciliarios; los estratos 5 y 6 corresponden a estratos altos que albergan a los usuarios con mayores recursos económicos, los cuales deben pagar sobrecostos (contribución) sobre el valor de los servicios públicos domiciliarios. El estrato 4 no es beneficiario de subsidios, ni debe pagar sobrecostos, paga exactamente el valor que la empresa defina como costo de prestación del servicio" [15]. 
- Mejoras en el acceso a la educación, aunque persisten desigualdades de acceso a la educación secundaria, técnica y superior entre niveles socioeconómicos, raza/etnia y zonas urbanas y rurales.

- El sistema de salud tiende a la universalidad del aseguramiento y a un paquete único de servicios, pero con desigualdades de acceso y calidad.
A partir de los relatos, los casos se clasificaron en 4 niveles (alto, medio, medio-bajo y bajo) por categorías, que reflejan las variaciones y semejanzas. En la Figura 1 se muestra la clasificación de los casos analizados, resaltando los extremos.

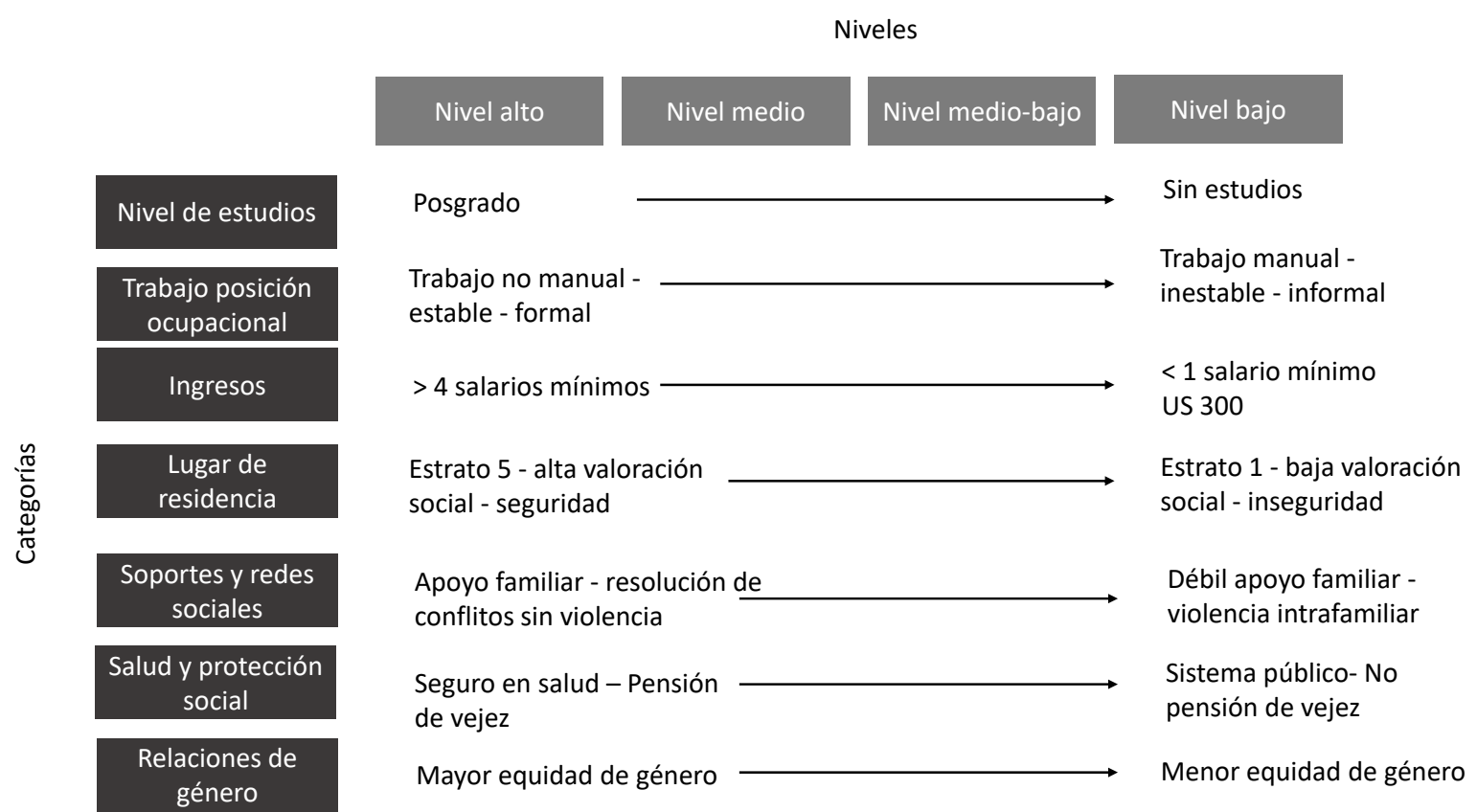

Figura 1. Clasificación de los participantes según niveles por categorías

En las trayectorias de vida, el encadenamiento que marcó diferencias entre niveles es: mayor nivel de escolaridad (capital cultural institucionalizado - títulos)-mayor posición ocupacional-mayor estabilidad y formalidad laboral-mayores ingresos (capital económico). Esto corresponde al índice de conversión, relacionado con los cambios en los tipos de capital a lo largo del tiempo [18]. En los niveles alto y medio, la inversión en educación durante la infancia y la juventud permitió el acceso a empleos de mejor calidad y mayores ingresos, en comparación con los otros niveles. En los niveles medio-bajo y bajo, aunque algunos continuaron sus estudios en la vida adulta, no consiguieron altos niveles de escolaridad ni una inserción laboral estable ni bien remunerada.

Frente a experiencias de manejo de la diabetes, a partir de este encadenamiento se observan diferencias entre quienes acceden a servicios de salud públicos o privados, y quienes tienen el paquete básico universal de servicios o quienes pueden acceder a servicios complementarios. Igualmente, entre quienes pueden sortear económicamente las contingencias de la enfermedad y quienes no lo pueden hacer $\mathrm{y}$, por tanto, ven afectadas su condición de salud y económica, o entre aquellos que pueden acceder a ciertos productos, alimentos o servicios para su cuidado (vía salario o pensión) y aquellos que no pueden costearlos.

Yo pesaba 87 kilos, pero ahora estoy como en 94 kilos. Yo camino y trato de cuidarme en los alimentos, pero pasa que no siempre tengo dinero para comprar los alimentos que debo comprar, como pan integral, arroz integral o fruta. Entonces, voy a la tienda, que ya me conocen, y fío arroz normal, porque ahí no venden integral; fío la harina para hacer la hojaldra; la leche normal, porque no venden descremada. O sea, como cosas que no debo. Quisiera seguir la dieta, pero no puedo, porque el arriendo que me pagan por las piezas que alquilo no siempre llega cumplido y debo pagar los servicios públicos, que son caros (Mujer, nivel bajo, entrevista, 2018).

Ligado a esta posibilidad de acceso a servicios y consumo, se articula el lugar de residencia como otro eslabón que marca un acceso desigual a bienes y servicios. Las relaciones entre urbano/rural o centro/periferia urbanos, determinan posibilidades diferenciales de 
disponibilidad de infraestructura de servicios de salud, deportiva y de recreación, así como limitaciones, debido a exposiciones diferenciales de riesgo. En los sectores de menores ingresos o periféricos, hay menos disponibilidad de servicios e infraestructura de uso público, así como de garantías de seguridad física. El territorio, en este sentido, se configura al mismo tiempo como producto y productor de desigualdades, afectando las formas de vivir, de enfermar y de cuidado [19].

Yo hago actividad física, porque trabajo cargando y descargando una camioneta; pero dicen que eso no es actividad física. Yo salía a un parque, pero una vez me iban a robar, y yo no volví por allá. Uno sale a trotar a las $5 \mathrm{a}$. $\mathrm{m}$. y no falta el que sale a robarle las chanclas (Hombre, nivel bajo, entrevista, 2018).

Las diferencias respecto al capital social también reflejan desigualdades. Por una parte, la densidad de las redes va a estar determinada por la condición socioeconómica de las familias, los tipos de relación y el manejo de los conflictos interpersonales. En la medida en que baja el nivel socioeconómico, se observan mayores niveles de violencia social e intrafamiliar, con mayor afectación de las mujeres. Las rupturas familiares por conflictos interpersonales, violencia o por razones económicas, junto con la destinación de tiempo para sobrevivir, reducen el tiempo para el cuidado mutuo.

Yo vivo con mi hija, pero ella trabaja todo el día, y a mí me toca hacer todo sola, el aseo de la casa, vendo bolsas de basura por encargo y caminando por el barrio. Hace tiempo vengo sintiendo dolor en las piernas y un pie se me inflama. El médico me mandó acetaminofén y me hice unos exámenes, pero no sé los resultados; el médico me dice que puede ser un problema circulatorio (Mujer nivel bajo, entrevista, 2018).

Por otra parte, los beneficios de las redes son desiguales. En los sectores de mayores ingresos, aunque existieron situaciones de violencia, se observa una tendencia a acumular capital social, debido a la menor disolución de las familias y de capital económico, a través de los hijos (mayor nivel de escolaridad, mejores ingresos), mayor cuidado de los padres por parte de sus hijos, al igual que mayor acceso a mecanismos de protección social, como planes de salud complementarios y pensión por vejez.

De la misma manera que mejoran las condiciones económicas y socioculturales de los casos, lo hacen sus contactos, generando así beneficios de cuidado directo y ganancias adicionales de bienestar, lo que facilita movilizar otros capitales por medio del capital social $[20,21]$. Una entrevistada señala:

Con alguna frecuencia, vamos a descansar a la finca de mi yerno, que queda en un clima muy bueno para mi esposo y para mí [...]. Mi hijo, que vive en Londres, me manda un endulzante que yo uso y con el que me siento muy bien (Mujer nivel alto, entrevista, 2018).
Sumando nuevos eslabones, es posible articular capitales de carácter simbólico que configuran las relaciones sociales y clasifican a ciertos individuos y prácticas como dominantes. En los relatos, se manifiesta un predominio del saber experto y de la figura del profesional de la salud sobre el saber y la experiencia de los pacientes. Aunque se evidenció en todos los niveles, se presenta con mayor intensidad en los niveles medio-bajo y bajo un desface entre las necesidades de los pacientes $\mathrm{y}$ las prescripciones del personal de salud, el predominio de una relación vertical, así como una deslegitimación de ciertos problemas y necesidades de los pacientes por la medicina, como muestran otros estudios con pacientes crónicos, aun cuando sean relevantes para ellos [22]. Un testimonio indica:

El médico y la gerontóloga que me ve ahora me dicen que debo hacer ejercicio y cumplir la dieta, pero nunca me preguntan si tengo plata para cumplirla y yo no les digo nada (Mujer nivel bajo, entrevista, 2018).

También se visibilizan otras diferencias, marcadas por los estereotipos de género. El rol de cuidadoras asumido/impuesto por las mujeres y las fracturas permanentes de las relaciones conyugales por violencia, sobre todo en los niveles medio-bajo y bajo, las obliga a asumir cargas económicas con baja capacidad para generar ingresos y menos tiempo para su cuidado personal. Las mujeres entrevistadas refirieron sobrepeso y altos niveles de estrés, que afectan su salud y el manejo de la diabetes, según ellas, debido a la sobrecarga de obligaciones afectivas y económicas, y de la confluencia de necesidades familiares insatisfechas y condiciones adversas durante sus trayectorias.

En consecuencia, el cuidado de sí por parte de las mujeres de niveles bajos se ve condicionado, a lo largo de su trayectoria de vida y particularmente frente al manejo de la diabetes, por el privilegio del cuidado de los otros en detrimento de sí misma, así como por la responsabilización por la provisión económica. Esto coincide con la afirmación que señala que, para las mujeres, el hecho de "convivir" significa invariablemente "cuidar" y no solo a los niños, sino de todas las personas que forman parte del hogar [23]. Otras afirman que "las mujeres pobres son doblemente pobres: en términos de ingresos y de tiempo" [24].

Otro eslabón que se articula a los eslabones materiales y simbólicos mencionados corresponde a las técnicas corporales [25] aprendidas a lo largo de la vida. El hecho de incorporar, en sus vidas prácticas, la actividad física cotidiana, un tipo de dieta o la necesidad de bajar de peso, para algunos resulta un cambio normal y consistente con su historia personal, mientras, para otros, se convierte en una novedad que requiere ser aprendida. Las posiciones económicas, la disposición y el acceso a alimentos o infraestructura deportiva, la presión social con respecto al cuidado y la apariencia física, la auto- 
percepción/práctica estética y de salud corporal, la disposición o no de tiempo, la relación entre el cuidado de sí y el cuidado de los otros, determina la facilidad y las formas como las personas asumen las prácticas prescritas, lo cual dista de ser simples decisiones personales.

\section{\#1 Propuesta teórico-metodológica}

Antes de continuar, es de advertir que este apartado busca aportar algunas categorías que contribuyan al desarrollo de análisis de desigualdades en salud desde abordajes cualitativos. Lo que se presenta a continuación resulta de articular los resultados presentados con el marco teórico que sustenta la investigación. En tal sentido, se proponen tres ámbitos diferenciados, que interactúan produciendo tipos de trayectorias de vida diferenciadas y desiguales, que configuran y se expresan en experiencias de padecimiento y de vida. El valor de esta propuesta frente a otras es su potencial para integrar diferentes aspectos de la realidad, que han sido analizados aisladamente en otros estudios (por ejemplo, aquellos que se centran en condiciones socioeconómicas [8]); además, se recurre a relatos biográficos, lo que contrasta con el predominio de fuentes cuantitativas y técnicas estructuradas, más frecuentes en los análisis de las desigualdades en salud.

De esta manera, de acuerdo con los resultados presentados, se identifican tres ámbitos de análisis que pueden orientar el estudio de las desigualdades en salud mediante abordajes cualitativos. Estos son: el ámbito de la salud, el ámbito socioeconómico/cultural y el contexto. Adicionalmente, están la infancia, la juventud, la adultez y la vejez, como etapas de análisis, basadas en la perspectiva de curso de vida de las desigualdades en salud, que postula que la salud de los individuos se produce a partir de la articulación de las biografías personales y la historia social en las vidas humanas [26,27]. En la Figura 2 se describe la estructura de la propuesta que se desarrolla a continuación.

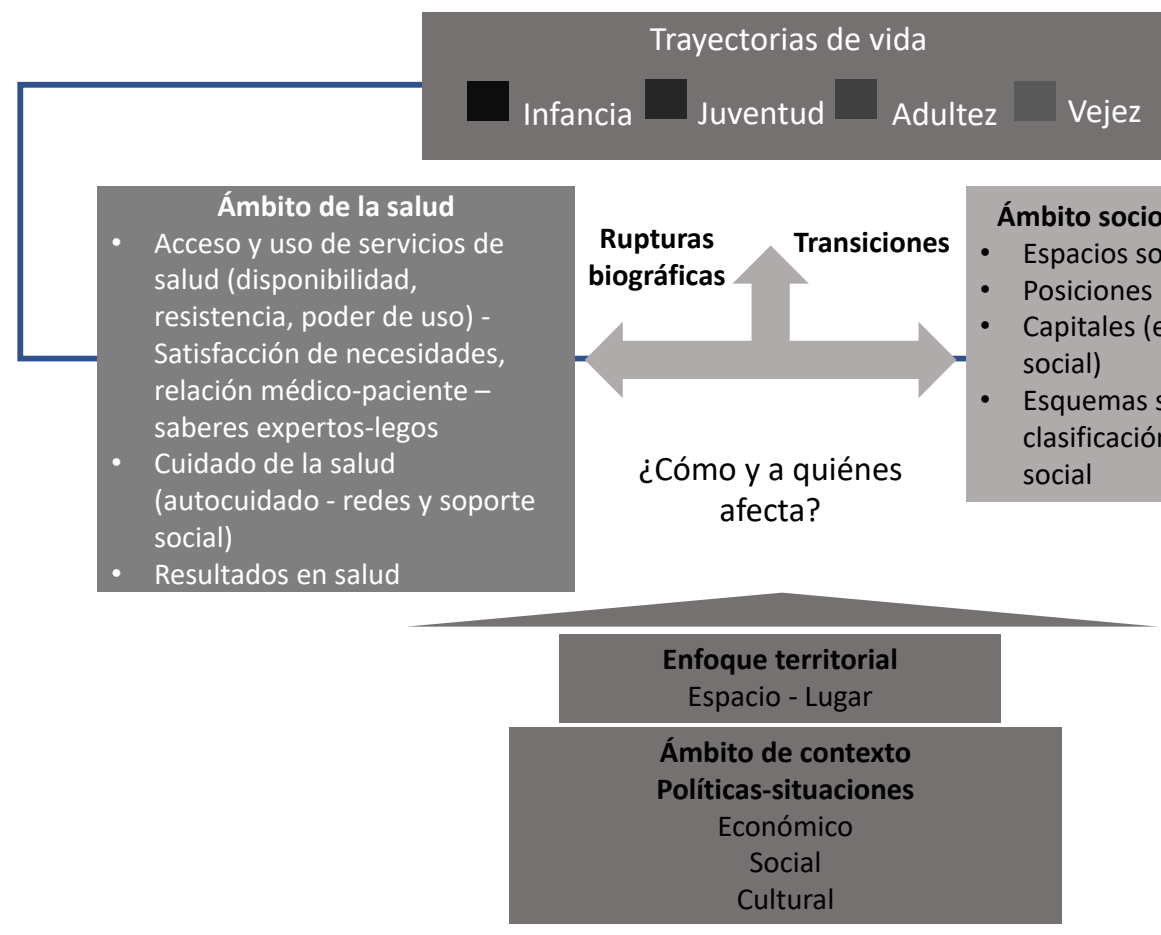

Figura 2 Herramientas para analizar las desigualdades en salud

En el primer ámbito de análisis, el ámbito de la salud, se identifican como nodos de articulación de procesos sociales e individuales y de desigualdades sociales y de salud: el acceso y el uso de los servicios de salud, el cuidado de la salud y los resultados en salud. En torno a estas articulaciones, no solo se evidencian las desventajas de uno sobre otros, sino también sus conexiones con aspectos de las trayectorias de vida. Se encontró que todos estos ámbitos están mediados por capitales culturales (educativo), sociales (redes, contactos), económicos (posición ocupacional, ingresos) y simbólicos (prestigio, reputación).

En esa medida, algunos modelos de acceso a servicios de salud ofrecen luces para este tipo de análisis. Una posible alternativa considera la relación entre la disponibilidad de los servicios, la resistencia y el poder de utilización. La disponibilidad se refiere a la existencia de los recursos físicos, humanos, administrativos y 
tecnológicos para prestar los servicios; la resistencia corresponde al conjunto de obstáculos para la búsqueda y la obtención de la atención (relacionadas directamente con los servicios o con el contexto), y el poder de utilización designa la capacidad de una persona o un grupo de personas para adquirir algún bien o servicio [28].

A esto se añade otra dimensión, relacionada con la satisfacción de las necesidades, y que fue uno de los hallazgos de este estudio. Al respecto, podría considerarse una dimensión que incluye las respuestas a las necesidades que el conocimiento específico en el tema valora como necesarias y que asume a todos como iguales (por ejemplo, controles periódicos de glicemia, controles médicos y nutricionales, entrega de medicamentos); así mismo, abarca las necesidades sentidas por los pacientes, que incluyen dimensiones emocionales, psicosociales y físicas, reconociendo sus diferencias [29].

Los resultados muestran una dimensión adicional, asociada con la relación entre médico y paciente, y entre saberes expertos y legos. En este sentido, aunque el capital cultural y económico pueden arrojar luces sobre posibles diferencias y desigualdades, algunos aportes de modelos explicativos, redes semánticas o sistemas de signos, significados y acciones, provenientes de la antropología médica [30], permiten explorar diferencias no solo desde una dimensión cultural, sino también como discursos que legitiman saberes médicos sobre experiencias de algunos pacientes [22] y que ponen en juego relaciones de poder, producto de las posiciones sociales entre personal de salud y los pacientes/clientes/ciudadanos, configurando relaciones verticales u horizontales $[31,32]$.

En relación con el cuidado de la salud, los resultados van en la misma línea de otros modelos de medición de desigualdades, que reconocen los comportamientos y los soportes sociales. Sin embargo, a diferencia de algunos modelos que conciben los comportamientos como conductas individuales, sujetos a la disponibilidad de información y a la conciencia sobre su salud, desde esta perspectiva se entienden las prácticas como producciones sociales que se modelan a lo largo de la vida, bajo condiciones de diferenciación y desigualdades sociales. El concepto de habitus, asociado a las técnicas corporales mencionadas en los resultados, es pertinente para este análisis, al referirse a la incorporación de lo social en la producción de la subjetividad, funcionando como esquemas de disposiciones duraderas que modelan las prácticas y los gustos de los diferentes grupos sociales. Detalles como la forma de hablar o la manera de mover el cuerpo, está inscrita en la posición social que ocupan los sujetos [33].

En cuanto a las redes, además de estudiar su densidad y los beneficios directos $[5,13,26]$, desde esta perspectiva se analizan durante las trayectorias de vida, procurando encontrar los procesos que las fortalecen o debilitan, y su relación con otros capitales, siguiendo la idea del índice de conversión mencionado en los resultados, es decir, capitales económicos y culturales que pueden generar capital social y viceversa. En ese sentido, se exploran los beneficios, las ganancias y los rendimientos del capital social, dada su capacidad de generar en provecho propio otros beneficios, como redes sociales movilizables [20,34].

Respecto a los resultados en salud, al mismo tiempo de los efectos tradicionalmente estudiados sobre la comorbilidad, la muerte prematura, la discapacidad, e inclusive los costos económicos de dichos efectos, bajo esta perspectiva se consideran las continuidades/rupturas entre vulnerabilidad-resultados en salud-vulnerabilidad. En las trayectorias de vida, se propone analizar los resultados en salud, articulados a las condiciones de vida (capitales, posiciones sociales) sobre las cuales son producidos, afrontados, superados, al igual que sus efectos en las condiciones de vida de los sujetos que los padecen. En otras palabras, deben tomarse en cuenta las condiciones sociales en que son producidos determinados resultados de salud y, al mismo tiempo, los efectos que estos generan. Con ello se pretende reconocer la multidimensionalidad económica, psicosocial y biológica de la vulnerabilidad social y los resultados en salud, referidos a un contexto social específico, ligados a las capacidades para ser, hacer, estar y tener.

El segundo ámbito de análisis, el ámbito socioeconómico/cultural, se refiere al tránsito de los sujetos por los principales espacios sociales, entendidos como sistemas de relaciones con funciones sociales específicas, códigos y normas formales e informales, que regulan su funcionamiento, donde los sujetos ocupan posiciones, definidas unas en función de otras, de acuerdo con sus capitales materiales y simbólicos [35]. En este estudio aparecen principalmente la familia de origen y de procreación o conyugal, los espacios educativos y los espacios laborales. No obstante, en cada análisis aparecerán estos u otros espacios, dependiendo de las trayectorias de los casos que estén siendo analizados.

En cada etapa de la vida aparecerán ciertos espacios como protagónicos y el análisis debe concentrarse en caracterizar el tipo de relaciones y las posiciones de los sujetos, en función de los capitales de los que dispone y de los esquemas socioculturales de clasificación/jerarquización de los individuos y sus atributos. A partir de ello, es posible identificar posiciones de desigualdad que, a su vez, marcan las posibilidades de tránsito a otros espacios sociales, así como su conexión con cada uno de los nodos del ámbito de la salud antes mencionados.

Por otra parte, respecto a las posiciones, más que un dato estadístico [4], en esta propuesta se analizan los procesos por los cuales se configuran relaciones posicionales en diferentes espacios sociales, como expresiones de diferenciación y desigualdad social. Las posiciones, 
entonces, antes de ser una cuestión del azar, son expresiones de relaciones de poder que estructuralmente clasifican a los sujetos, y de disputas relacionales que las reproducen y transforman.

Así, en las familias se encuentran posiciones de dominación/sumisión/discriminación según género, si se es hijo dentro del matrimonio o fuera de este, o si se gana más dinero en las relaciones conyugales. Esas posiciones van a determinar el acceso a la educación de los hijos, o a trabajos remunerados, en el caso de las mujeres. En los espacios educativos, a su vez, puede haber posiciones de ventaja/desventaja, según género, condición étnico racial, círculo de amigos (capital social), prestigio familiar, capital económico de la familia o el lugar de residencia. En los espacios laborales, la posición ocupacional va a estar determinada por el tipo de cargo (manual/intelectual, directivo/asistente/operario), el volumen de capital recibido (capital económico), que, en gran medida, va a estar mediado por combinaciones entre capital cultural adquirido — vía títulos-, capital económico — propio o adquirido por la trayectoria familiar-, capital social y prestigio social [18].

De los resultados presentados, se extraen adicionalmente los conceptos de transiciones y rupturas biográficas. Las transiciones se refieren a los cambios de estado, posición o situación que tienen durante las trayectorias de vida. Ello marca el tránsito hacia nuevos espacios sociales y, por consiguiente, a nuevos roles, derechos, obligaciones y facetas de identidad social. Algunas de las transiciones ocurren de manera secuencial, ajustadas al sistema de expectativas socialmente establecido; otras se manifiestan simultáneamente y, además, en momentos de la vida no previstos [27]. Algunos ejemplos del presente estudio son las entradas y salidas del sistema educativo en la niñez, la juventud y la adultez, el ingreso al mercado laboral a edades tempranas, la entrada y la salida de relaciones conyugales, la jubilación y las migraciones forzadas.

Las rupturas biográficas, por su parte, corresponden a eventos favorables o desfavorables que provocan impacto y modificaciones que, a su vez, se traducen en virajes en la dirección del curso de vida y, por ende, en transiciones [27]. A diferencia de las transiciones, que pueden tener algún grado de previsibilidad, las rupturas biográficas son intempestivas, generan una alta carga emocional y son capaces de producir efectos incontrolables para quien las padece, en términos económicos, socioafectivos y de salud física y psicológica. En los casos analizados, se referencian, como rupturas biográficas, la muerte de familiares, diagnósticos de enfermedades, crisis económicas, rupturas familiares, hechos que por cierto algunos relacionan con la aparición de la diabetes.

El contexto, como tercer ámbito de análisis, si bien mantiene cierto carácter de exterioridad con los casos analizados, a diferencia de otros modelos, en este, emer- ge de las trayectorias de vida analizadas. Se identifican, entonces, situaciones de la historia social relacionadas con decisiones políticas y transformaciones económicas, educativas, dinámicas de violencia social y política, como las que se registraron en este trabajo. Para organizar su análisis, metodológicamente puede ser pensado bajo una perspectiva territorial, que diferencia hechos que involucran a todos los habitantes de un sector, o que lo hacen de manera relativa, de acuerdo con su ubicación y su dinámica.

A propósito de esa distinción, se propone diferenciar entre espacio y lugar, como lo hacen otros modelos de medición de desigualdades [26]. El espacio tiene que ver con la distancia o proximidad de los hechos al sujeto analizado, así como la intensidad y la distribución de estos, geográfica y socialmente. Por ejemplo, la ubicación de zonas de conflicto en las zonas rurales, hechos de inseguridad urbana, de infraestructura física o educativa, o la distribución de mercados que ofrecen ciertos alimentos. El lugar, en cambio, se refiere a la afiliación a unidades políticas o administrativas, cuyas políticas o programas operan uniformemente dentro de sus límites, independientemente de la ubicación física de los residentes; por ejemplo, programas de asistencia alimentaria, políticas tributarias o educativas.

\section{Conclusiones}

Según los resultados y para los estudios de las desigualdades de otras patologías, se identifican cuatro ejes claves en la producción de desigualdades sociales/de salud, que aparecen articulados en la propuesta de análisis. Tanto en las trayectorias de vida como en las experiencias de enfermedad, el territorio es un eje estructurador. Determina posiciones y oportunidades de acceso a servicios, a la tierra, la vivienda, la educación, el trabajo de las familias, así como imaginarios sobre sí mismos y de los otros. Igualmente, marca la exposición a riesgos y la disponibilidad/uso a infraestructura, al tiempo que condiciona formas de pensar, imaginar y actuar.

El vínculo educación-trabajo también determina las posiciones de ventaja y desventaja social. Refleja la articulación entre mayor capital humano a través de la educación, la calidad del trabajo, el nivel de ingresos y otros beneficios. Se constituye en punto de inflexión diferencial entre niveles, en términos de movilidad social biográfica e intergeneracional.

De manera transversal, el sistema de género condiciona las relaciones, posicionando al hombre por encima de la mujer. Marcó, así, la configuración de esquemas de roles sociales durante la socialización, y aunque estos esquemas fueron objeto de disputa en los escenarios sociales donde transcurre la vida de los sujetos, prevalecieron las formas hegemónicas de desigualdad (lo mas- 
culino sobre lo femenino), en las relaciones familiares, la educación, el trabajo y el cuidado de sí mismo y de los demás. Se evidenció una relación de desventaja de las mujeres, al igual que entre mujeres de diferentes niveles.

De igual modo, la violencia política afecta principalmente las zonas rurales, generando migraciones forzadas y perturbaciones económicas, educativas, afectivas y culturales. La violencia social de contextos urbanos, por su parte, provocó trastornos biográficos que limitaron el desarrollo de capacidades y el acceso a bienes y servicios. Finalmente, la violencia familiar atravesó la vida, especialmente de las mujeres, restringiendo su acceso a la educación, el trabajo, sus vínculos afectivos y las relaciones consigo mismas.

\section{Fuente de financiación}

Recursos propios.

\section{Conflicto de interés}

El autor declara no tener relación personal, científica, comercial o financiera con personas o instituciones que pudieran sesgar los resultados presentados.

\section{Declaración de responsabilidad}

Declaro que los puntos de vista expresados son responsabilidad del autor y no de la institución en la que trabaja.

\section{Agradecimientos}

Agradezco a la profesora Rita de Cássia Duarte Lima, de la Universidade Federal do Espírito Santo (Brasil), por sus aportes a la escritura de este artículo.

\section{Referencias}

1. Barreto M. Desigualdades em Saúde: uma perspectiva global. Ciênc. Saúde Coletiva. DoI: https://doi.org/10.1590/141381232017227.02742017

2. Correa AM, Arias MM, Carmona J. Equidad e igualdad sociales y sanitarias. Necesidad de un marco conceptual científico. Medicina Social [internet]. 2012 [citado 2019 jul. 12]; 7(1):5-13. Disponible en: https://www.medicinasocial.info/index.php/medicinasocial/ article/view/571

3. Fiorati RC, Arcêncio RA, Souza LB. As iniquidades sociais e o acesso à saúde: desafios para a sociedade, desafios para a enfermagem. Rev. Latino-Am. Enfermagem. 2016;24:e2683 Dor: https:// doi.org/10.1590/1518-8345.0945.2687

4. Mújica OJ, Moreno CM. De la retórica a la acción: medir desigualdades en salud para "no dejar a nadie atrás". Rev Panam Salud Publica. 2019;43:e12. Dor: https://doi.org/10.26633/RPSP.2019.12
5. Mújica OJ. Cuatro cuestiones axiológicas de la epidemiología social para el monitoreo de la desigualdad en salud. Rev Panam Salud Publica [internet]. 2015 [citado 2019 ago. 3]; 38(6):433-41. Disponible en: https://pdfs.semanticscholar.org/85c6/9f1848d844 dd9766625b79f5744ea489a01b.pdf

6. Santana P, Costa C, Loureiro A, et al. Geografias da Diabetes Mellitus em Portugal: como as condições do contexto influenciam o risco de morrer. Acta Med Port [internet]. 2014 [citado 2019 jul. 20]; 27(3):309-17. Disponible en: https://pdfs.semanticscholar. org/d41a/b5445c21d04e555683571ba31e1 feb2e423e.pdf

7. Domínguez E. Desigualdades sociais e diabetes mellitus. Rev. Cubana Endocrinol. [internet]. 2013 [citado 2019 ago. 10]; 24(2):20013. Disponible en: http://scielo.sld.cu/scielo.php?script=sci_artte xt\&pid=S1561-29532013000200009

8. Wu H, Meng X, Wild S, et al. Socioeconomic status and prevalence of type 2 diabetes in mainland China, Hong Kong and Taiwan: A systematic review. J Glob Health. 2017; 7(1):1-14. DoI: https:// doi.org/10.7189/jogh.07.011103

9. Johnson JA, Cavanagh $\mathrm{S}$, et al. The diabetes disparity and Puerto Rican identified individuals: A systematic literature review. Diabetes Educ. 2017;(43)2: 153-62.ago. Dor: https://doi. org/10.1177/0145721716687662

10. Cerezo-Correa M, Cifuentes-Aguirre O, et al. Desigualdades de la morbilidad por enfermedades crónicas según determinantes estructurales e intermediarios. Rev. Gerenc. Polit. Salud [internet]. 2012 [citado 2019 jul. 15]; 11(23):165-88. Disponible en: https:// www.redalyc.org/pdf/545/54525297012.pdf

11. Cali Cómo Vamos. Informe anual 2018 de calidad de vida Cali. Santiago de Cali; 2018.

12. Riley D. A teoria das classes de Pierre Bourdieu. Estud. Sociol. [internet]; 2019 [citado 2020 ene. 15]. 24(46):181-210. Disponible en: https://periodicos.fclar.unesp.br/estudos/article/ view/12245/8390

13. Arias S. Epidemiología, equidad en salud y justicia social. Rev. Fac. Nac. Salud Pública. 2017;35(2):186-96. DoI: https://doi. org/10.17533/udea.rfnsp.v35n2a03

14. Ministerio de Salud y Protección Social de Colombia. Aseguramiento al Sistema General de Seguridad Social en Salud. Bogotá; 2014. https://www.minsalud.gov.co/sites/rid/Lists/BibliotecaDigital/RIDE/VP/DOA/RL/cartillas-de-aseguramiento-al-sistemageneral-de-seguridad-social-en-salud.pdf

15. Departamento Administrativo Nacional de Estadística (DANE). Preguntas frecuentes estratificación [internet]. s. f. [citado 2019 jul. 15]. Disponible en: https://www.dane.gov.co/files/geoestadistica/Preguntas_frecuentes_estratificacion.pdf

16. Díaz C. Investigación cualitativa y análisis de contenido temático. Orientación intelectual de revista Universum. Rev. Gen. Inf. Doc. 2018;28(1):119-42. DoI: https://doi.org/10.5209/RGID.60813

17. Aigneren, M. Análisis de contenido: una introducción. La sociología en sus escenarios [internet] 1999 [citado 2019 ago. 02]; (3):152. Disponible en: https://revistas.udea.edu.co/index.php/ceo/ article/view/1550

18. Weininger E. Fundamentos de uma análise de classe de Pierre Bourdieu. Em: Wrigth E, organizador. Análise de classe. Abordadens. Petrópolis, Brasil: Editora Vozes; 2015.

19. Borde E, Torres M. El territorio como categoría fundamental para el campo de la salud pública. Saúde em debate. 2017;41(núm. especial):264-75. Dor: https://doi.org/10.1590/0103-11042017S222

20. Pena JA, Sánchez JM. El capital social individual: lo micro y lo macro en las relaciones sociales. En: José Luis Veira Veira, coor- 
dinador. Desigualdad y capital social en España. Oleiros: Netbiblo; 2013. pp. 11-41

21. Bourdieu P. Poder, derecho y clases sociales. Bilbao: Desclee de Brouwer; 2001.

22. Canesqui AM. Legitimidade e não legitimidade das experiências dos sofrimentos e adoecimentos de longa duração. Ciênc. Saúde Colet. 2018;23(2):409-16. Dor: https://doi.org/10.1590/141381232018232.14732017

23. Rohlfs I, Borell C, Fonseca MC. Género, desigualdades y salud pública: conocimientos y desconocimientos. Gac. Sanit. [internet]. 2000 [citado 2019 jul. 30]; 14(3):60-71. Disponible en: http://www.gacetasanitaria.org/es-genero-desigualdades-saludpublica-conocimientos-articulo-X0213911100956185

24. Cecchini S, Madariaga A. Programas de transferencias condicionadas. Balance de la experiencia reciente en América Latina y el Caribe. Cuadernos Cepal 95 [internet]; 2011 [citado 2019 jul. 24]. Disponible en: https://repositorio.cepal.org/bitstream/handle/11362/27854/S2011032_es.pdf

25. Calle Valverde J. de la. El gesto analógico. Una revisión de las "técnicas del cuerpo" de Marcel Mauss. Revista Latinoamericana de Estudios sobre Cuerpos, Emociones y Sociedad [internet]. 2011-2012 [citado 2019 jul. 25]; 3(7):75-87. Disponible en: http:// www.relaces.com.ar/index.php/relaces/article/viewArticle/126

26. Arcaya MC, Arcaya AL, Subramanian SV. Inequalities in health: Definitions, concepts, and theories. Glob Health Action. 2015;8(1):27106. DoI: https://doi.org/10.3402/gha.v8.27106

27. Blanco M. El enfoque del curso de vida: orígenes y desarrollo. Revista Latinoamericana de Población [internet] 2011 [citado 2019 jul. 25]; 5(8):5-31. Disponible en: https://www.redalyc.org/ pdf/3238/323827304003.pdf
28. Frenk J. El concepto y la medición de la accesibilidad. Salud Pública Méx [internet]. 1995 [citado 2019 jul. 26]; 27:438-53. Disponible en: http://www.saludpublica.mx/index.php/spm/article/ view/422/411

29. Arrivillaga M, Borrero Y. Visión comprensiva y crítica de los modelos conceptuales sobre acceso a servicios de salud, 19702013. Cad. Saúde Pública, [internet]. 2016 [citado 2019 jun. 25]; 32(5):1-15. Disponible en: http://www.scielo.br/pdf/csp/ v32n5/1678-4464-csp-32-05-e00111415.pdf

30. Uchôa E, Vidal J. Antropologia médica: elementos conceituais e metodológicos para uma abordagem da saúde e da doença. Cad. Saúde Públ. 1994;10(4):497-504. DoI: https://doi.org/10.1590/ s0102-311x1994000400010

31. Ayuzo del Valle C. Pacientes, clientes, médicos y proveedores, ¿es solo cuestión de terminología? Gac Med Mex [internet]. 2016 [citado 2019 jun. 22]; 152:429-30. Disponible en: https://www. anmm.org.mx/GMM/2016/n3/GMM_152_2016_3_429-430.pdf

32. Lemus S, Hamui A, et al. Una mirada crítica sobre la noción: paciente/usuario/cliente desde la antropología en salud. Revista CONAMED. [internet]. 2017 [citado 2019 ago. 10]; 22(2):98-103. Disponible en: https://www.medigraphic.com/cgi-bin/new/resumen.cgi?IDARTICULO $=79307$

33. Bourdieu P. La distinción. Críticas y bases sociales del gusto. Madrid: Taurus; 1998.

34. Capdevielle J. Capital social: debates y reflexiones en torno a un concepto polémico. Rev. Sociol. Polit. 2014;22(51):3-14. DOI: https://doi.org/10.1590/1678-987314225101

35. Bourdieu P. El espacio social y la génesis de las "clases". Estudios sobre las Culturas Contemporáneas [internet]. 1989 [citado 2019 jul. 22]; 3(7):27-55. Disponible en: https://www.redalyc.org/ pdf/316/31630703.pdf 